\title{
Introduction to ECSCW 2017
}

\author{
Charlotte P. Lee ${ }^{1}$, Luigina Ciolfi ${ }^{2}$ (D) \& David Randall ${ }^{3}$ \\ ${ }^{1}$ University of Washington, Seattle, WA , USA $;{ }^{2}$ C3RI, Sheffield Hallam University, 153 Arundel Street, \\ Sheffield, S12NU, UK (E-mail: L.Ciolfi@shu.ac.uk); ${ }^{3}$ University of Siegen, Siegen, Germany
}

This special issue of JCSCW is a collection of the long papers presented at ECSCW 2017, the 15th European Conference on Computer-Supported Cooperative Work, held from August 28 to September 1, 2017 in Sheffield (UK).

ECSCW is a series of international conferences on computer supported cooperative work located in Europe and championed by EUSSET, the European Society for Socially Embedded Technologies. For the first time in its history, ECSCW partnered with the CSCW Journal for the selection of long papers submitted to the conference, soliciting journal-quality contributions and adopting a journal-level review process for the submission of papers documenting original, rich, and in-depth contributions to $\mathrm{CSCW}$.

The ECSCW conference is an important venue for defining and developing the CSCW research agenda, focusing on better understanding the practices of cooperative work and on exploring the design of systems to support cooperative work. The conference has a longstanding interest in empirical, conceptual, and theoretical contributions and has a tradition of inclusiveness. The solicited themes included, but were not limited to:

- Conceptualising practice in work and other activities, and the relationship between understanding practice and the design of computer artifacts: how can we understand work (the "W")?

- Cooperation and its characteristics (e.g., describing the particulars of articulation work and coordination mechanisms in a given setting): how can we understand cooperative work (the "CW")?

- Methods for investigating human practices: the nature of ethnography and the role of other innovative methods in $\mathrm{CSCW}$;

- Digital and other material artefacts in cooperative settings: how can we support cooperative work in increasingly complex, networked settings (the "CS")?

It is worth noting that, in addition to the presentation of the long papers that are included in this special issue, the ECSCW 2017 conference also included a lively 
program of exploratory papers, workshops, masterclasses, panels, and demonstration sessions enabling broader and more active participation.

The conference received 52 long paper submissions that were reviewed by the the ECSCW program committee (PC) that comprised 85 members. Decisions were based on peer-reviews and were made by the PC chairs (the guest editors of this special issue) in collaboration with the Journal's Editor-In-Chief. The journal process for the ECSCW 2017 special issue involved one full review cycle and a second, correctionsconfirmation cycle. The process was compressed into a shorter amount of time than is typical for the Journal thus requiring a substantial effort from the PC members.

Seventeen papers were ultimately accepted and are presented in this special issue. These papers represent a wide range of CSCW topics and concerns and they substantially contribute to the tradition of in-depth studies of human practices mediated by digital interactive technologies that ECSCW has championed throughout its history. Topics covered include: designing for children, supporting local interactions, collaborative accounts and storytelling, studies of scientific work, money and finances, online working, and methods for civic engagement.

The ECSCW 2017 conference committee and the ECSCW community as a whole did suffer a huge loss during the planning process. As we set out to work on the scientific and technical programme, we lost one of our most respected, prolific and enthusiastic members. The late Dr. David Martin was holding the role of papers cochair for ECSCW 2017 when he suddenly passed away in June 2016. We wish to acknowledge his contribution to this special issue and to the conference, as well as to the ECSCW community overall. We still mourn him and he will be greatly missed.

From the darkness we have some light: His former institution, Xerox Research Center France, has generously established the "David B. Martin Best Paper Award" in his memory. The Award will be bestowed yearly upon a full paper accepted to ECSCW that particularly contributes to the multidisciplinary understanding of society and/or work from a CSCW perspective. While all accepted papers delivering such a contribution will be eligible for the award, preference will be given to papers authored or co-authored by early and mid-career researchers (max 10 years since $\mathrm{PhD}$ ). The inaugural Award will be formally presented to the winning paper at the ECSCW 2017 conference in Sheffield.

Finally, we wish to acknowledge the contributions of all the members of the ECSCW 2017 Programme Committee and Conference Committee who have worked so admirably under a tight time frame. We also acknowledge the support and advice of the Journal of CSCW Editor-In-Chief Kjeld Schmidt and of the CSCW Journal Editorial Office, including Lester de Leon, who had to deal "on the fly" with a completely new process and requirements. 